\title{
Null-induced mode changes in PSR B0809+74
}

\author{
A. G. J. van Leeuwen ${ }^{1}$, M. L. A. Kouwenhoven ${ }^{1}$, R. Ramachandran ${ }^{2,3}$, \\ J. M. Rankin ${ }^{2,4}$, and B. W. Stappers ${ }^{3}$ \\ 1 Astronomical Institute, Utrecht University, PO Box 80000, 3508 TA Utrecht, The Netherlands \\ 2 Astronomical Institute "Anton Pannekoek", Kruislaan 403, 1098 SJ Amsterdam, The Netherlands \\ 3 Stichting ASTRON, PO Box 2, 7990 AA Dwingeloo, The Netherlands \\ 4 Physics Department, University of Vermont, Burlington, VT 05405, USA
}

Received 17 December 2001 / Accepted 14 February 2002

\begin{abstract}
We have found that there are two distinct emission modes in PSR B0809+74. Beside its normal and most common mode, the pulsar emits in a significantly different quasi-stable mode after most or possibly all nulls, occasionally for over 100 pulses. In this mode the pulsar is brighter, the subpulse separation is less, the subpulses drift more slowly and the pulse window is shifted towards earlier longitudes.

We can now account for several previously unexplained phenomena associated with the nulling-drifting interaction: the unexpected brightness of the first active pulse and the low post-null driftrate. We put forward a new interpretation of the subpulse-position jump over the null, indicating that the speedup time scale of the post-null drifting is much shorter than previously thought. The speedup time scale we find is no longer discrepant with the time scales found for the subpulse-drift slowdown and the emission decay around the null.
\end{abstract}

Key words. stars: neutron - pulsars: general - pulsars: individual: PSR B0809+74

\section{Introduction}

Lately, it has been quiet around bright PSR B0809+74, once the canonical example of regular subpulse drifting and the centre of lively discussions.

In the thirty years since their discovery by Drake \& Craft (1968), drifting subpulses feature in many discussions about the nature of pulsars and the pulsar's emission mechanism. The subpulse-drifting phenomenon in itself is simple: when comparing adjacent individual pulses, the subpulses that comprise them are seen to shift regularly through the average pulse window. The left panel of Fig. 2, for example, shows a recent observation in which these subpulses form their driftbands.

In 1968, the interpretation of Drake \& Craft that the subpulse drifting is the pulsation of the neutron star, is ground zero for much debate. Over the following years, the ever increasing quality and volume of observations of subpulses in different pulsars continue to limit possible models and require their refinement. It is in this process that observations of $0809+74$ take the lead.

Alexeev et al. (1969) and Vitkevich \& Shitov (1970) are the first to detect the driftbands of $0809+74$. Cole (1970) then notes that the driftrate of the subpulses occasionally changes, without being able yet to identify the nulls as the trigger. One year later, Taylor \& Huguenin (1971) do

Send offprint requests to: A. G. J. van Leeuwen, e-mail: a.g.j.vanleeuwen@astro.uu.nl find that this occasional cessation of the pulsar's emission precedes a changing driftrate, lasting a few driftbands.

In his paper solely devoted to the drifting in $0809+74$, Page (1973) finds that the subpulse separation changes through the profile. He also introduces the "subpulse phase", the difference between the actual position of the subpulse and its predicted position. While this makes it easier to plot long series of subpulse positions horizontally, it also introduces the notion that the subpulse position dramatically jumps in phase over a null. From the data however, it is clear that the subpulse position over the null does not change much at all - on the contrary, it is the preservation of phase that is the astonishing phenomenon.

Unwin et al. (1978) are the first to note this. They find that the position of the subpulses is identical before and after the null and conclude that, even though there is no emission from the pulsar, the emitting structures survive the null. Five years later, Lyne \& Ashworth (1983) use a method devised by Ritchings (1976) to find the nulls of $0809+74$ and investigate the time scales associated with the change in drift pattern around a null. The first time scales they look into are those of the decay and rise of emission around a null. A finite decay and rise time would cause the pulses neighbouring a null to be less bright than the average. A relative dimness of the last pulse before the null is found indeed, pointing at a decay time shorter than $5 \%$ of the pulse period. Contrary to the expectations, however, the first pulses after the nulls are found to outshine the average ones. 
The next time scales considered are those of the slowdown and speedup of the subpulse drift around the null. Lyne \& Ashworth notice that, contrary to the findings of Unwin et al., there is a some change in subpulse positions over the null. They suggest that this change originates in subpulse drift that is caused by a gradual speedup of the drifting during the null. Being several tens of pulse periods, this speedup time scale is much larger than the time scales found for the emission decay and rise, and the subpulse drift slowdown.

In 1984, simultaneous observations at 102 and $1412 \mathrm{MHz}$ by Davies et al. confirm the curvature of the driftbands found by Page (1973) and show that the subpulse width varies across the pulse profile.

Although work on $0809+74$ quiets down after this intense period, significant observational progress for the understanding of subpulse drifting in general is made on 0943+10. Deshpande \& Rankin $(1999,2001)$ are able to detect periodicities in the subpulse strengths that lead to a determination of the recurrence time of individual subpulses.

On the theoretical front, the initial hypothesis that the subpulse drifting originates in pulsations of the neutron star (Drake \& Craft 1968) is soon abandoned. The subpulses are now thought to be formed in the magnetosphere of the pulsar, and the topic of debate changes to the exact location of their formation. The observed periodicity is first thought to be caused by quasi-periodic fluctuations in the plasma near the co-rotation radius of the pulsar. The polarisation and beaming of the subpulses, however, later tie the emission mechanism to the surface of the pulsar.

In 1975, Ruderman \& Sutherland build on the Goldreich \& Julian (1969) and Sturrock (1971) models to propose a theory that incorporates the drifting subpulses and the nulling. They suggest that the pulsar emission is formed in discrete locations around the magnetic pole. These locations would rotate around the pole like a carousel. In one of many revisions of the Ruderman \& Sutherland model, Filippenko \& Radhakrishnan (1982) propose a model that explains the survival of the subbeam structure over the null. The work of Deshpande \& Rankin $(1999,2001)$ visualises these models by mapping the observed subpulses onto their original positions on the carousel.

In this paper we investigate the behaviour of the pulsar emission around nulls. We will use observations of two extraordinary long sequences of post-null behaviour to explain the features of general subpulse drifting around nulls in a new framework.

\section{Observations}

\subsection{Data}

Over a period of about 18 months we have observed 0809+74 with PuMa, the Pulsar Machine, at the Westerbork Synthesis Radio Telescope (WSRT) in the

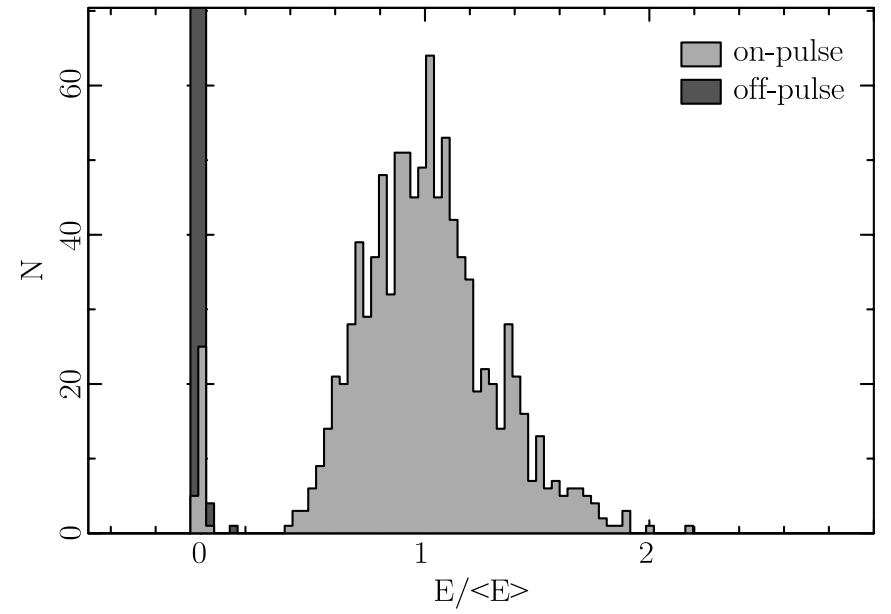

Fig. 1. Histogram of the energy in 1000 consecutive pulses, each scaled with the average energy of the 100 pulses that surround it. In the foreground (light gray) we show the distribution of the energies found in the on-pulse window. In the background (dark gray) the energies found in an equally large section of the off-pulse window are shown, peaking far off the scale around $N=900$.

Netherlands. Most observations lasted several times the scintillation time scale of this pulsar. We have only used the data in which the pulsar was bright, amounting to a total of 13 hours. With a pulse period of $1.29 \mathrm{~s}$, this comes down to $3.6 \times 10^{4}$ pulses.

The collecting area of WSRT and the low system noise made it possible for PuMa to record the data with a high timing resolution and a high signal-to-noise ratio (SNR). The WSRT consists of fourteen 25-meter dishes arranged in east-west direction. For our observations, signals from all the fourteen dishes were added after compensating for the relative geometrical delay between them, so that the array could be used like a single dish with an equivalent diameter of 94 meters. The pulsar was observed with PuMa (Kouwenhoven 2000; Voûte 2001; Voûte et al. 2002) at centre frequencies between $328 \mathrm{MHz}$ and $382 \mathrm{MHz}$, with bandwidths of $10 \mathrm{MHz}$. Each 10-MHz band was split into 64 frequency channels, and the data was recorded, after being digitised to represent each sample by four bits (sixteen levels). After inspection for possible electrical interference, we corrected for the interstellar dispersion during our off-line analysis.

\subsection{Reduction}

\subsubsection{Finding nulls}

Normally, the energy of individual pulses varies from about 0.5 to 2 times the average. When the pulsar is in the null state however, which it is for about $1.4 \%$ of the time, it is does not emit. In this case, very little energy is found where the pulse is expected. We have used this difference in on-pulse energies to identify the nulls. To do this, we compared the on-pulse energies with the energy found in off-pulse region, where no emission is expected. 

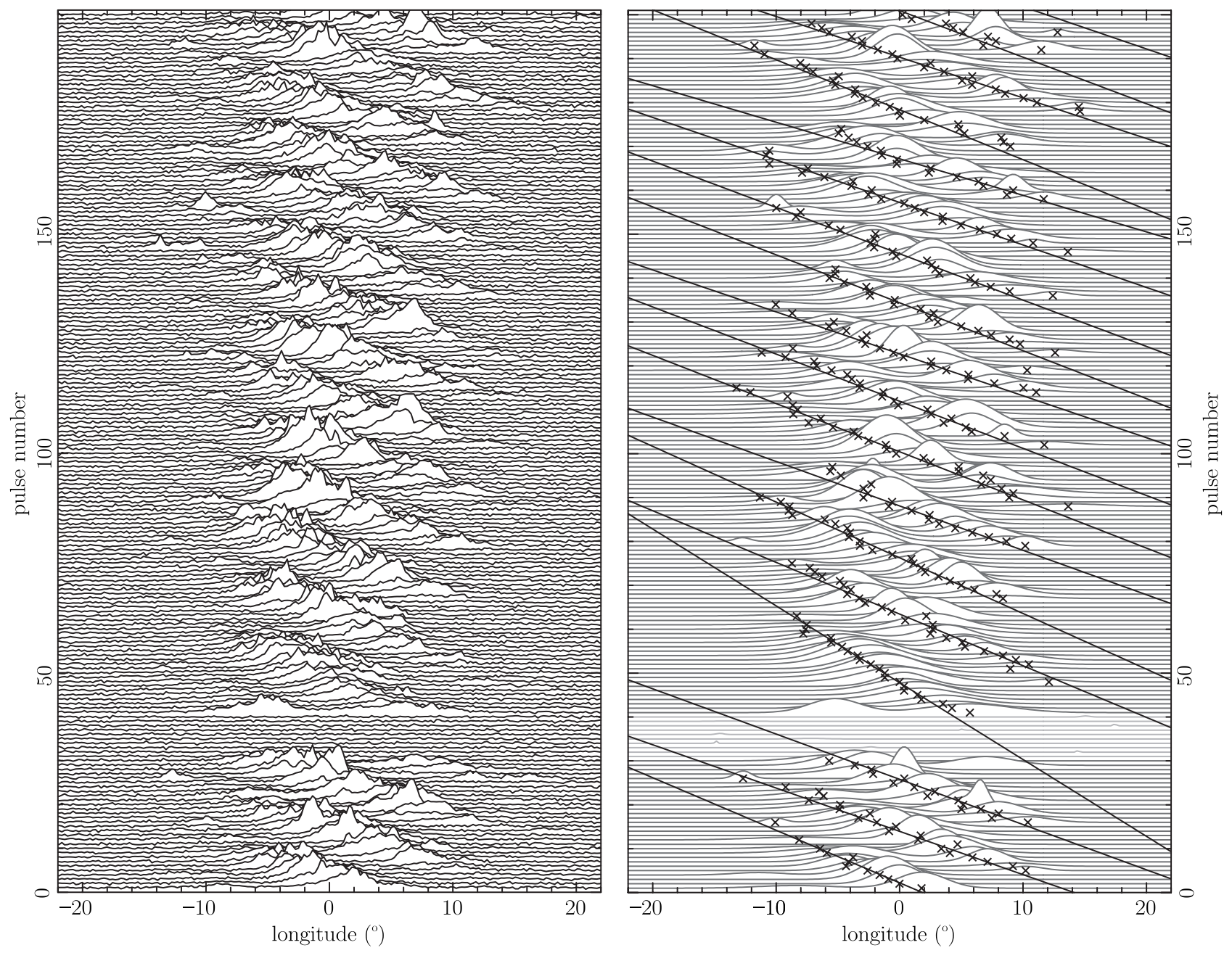

Fig. 2. Example of the fitting of the subpulses. Left panel: stacked pulses in the on-pulse region. Right panel: after finding nulls (lighter colour), and fitting subpulses (crosses at the base of the fitted Gaussians), we fit the driftbands (black lines). In this and all other figures, $0^{\circ}$ longitude is defined as the centre of the best Gaussian fit to the average profile.

To calculate the on-pulse and off-pulse energies, we split each pulse period $P_{1}\left(360^{\circ}\right.$ in longitude) into three sections. The first section, $0.15 P_{1}$ wide, was centred around the peak of the average profile. The second section, equally wide, consisted of a part of the off-pulse region. A third section, $0.5 P_{1}$ wide, contained a different, independent part of the off-pulse data and was used to estimate the noise and baseline of the signal. This baseline was subtracted from both the on-pulse and off-pulse data.

To calculate the energy in each pulse, we determined the central range in longitude that on average contained $90 \%$ of the power the pulsar emitted in the on-pulse section. For this region we summed the amplitudes of the signal to get the energy content. We did the same for an equally large piece of the off-pulse section. To correct for long term variations in the pulse energy due to scintillation, we subsequently scaled both energies with the average energy of the 100 surrounding pulses.

We defined that, in the null state, there is no observable radiation from the pulsar. This means that noise is all one observes at the time a new pulse is expected. The energy distribution of the null-state is then identical to that of the off-pulse region (high distribution in the background of Fig. 1). For our data this meant that all pulses with energies less than the highest energy found in the off-pulse distribution were nulls (small peak around zero energy in Fig. 1). Furthermore, the off-pulse distribution gives a better estimate of the expected spread in null energies than the null distribution itself, due to the larger number of pulses included.

This method, originally devised by Ritchings (1976), was first used on $0809+74$ by Lyne \& Ashworth (1983). In their case, there was considerable overlap between the on-pulse and off-pulse distributions, hindering their identification of the nulls. We wanted to be certain that the set of nulls we found was genuine and complete; therefore we only used the $60 \%$ of our observations in which the onpulse and off-pulse distribution were separated by more than $0.1 E /<E>$.

With a 3 -sigma point at $15 \mathrm{mJy}$, the low noise makes it possible to fully distinguish between pulses in the normal state on the one side, and those in the null state on the other, giving unprecedented insight into the nature of the null state. 


\subsubsection{Fitting subpulses}

The major step in our data reduction was to extract the four basic elements of the individual pulses: the number of subpulses and their respective positions, widths and heights. By doing so we greatly increased the speed of subsequent operations, but at the same time kept a handle on the physics by retaining the parameters most important for visualising the data.

The high SNR of our observations showed a wealth of microstructure in the individual subpulses, which on average were Gaussian in shape. Plainly fitting Gaussians to the intricate, many featured subpulses did not immediately result in locating them reliably. Due to the changing amplitude of the signal throughout the window, the microstructure of the brightest subpulses was often more important to the goodness-of-fit than complete, weaker subpulses near the edge of the pulse window. In order to still find these weak subpulses, we devised a two-step approach of first finding the strong subpulses and then using their driftpath to suggest the position of weaker subpulses. This approach worked very well.

For our fitting, we assumed the subpulses to be Gaussian in shape, non overlapping, and to have a full width at half maximum ( $F W H M$ ) less than $15^{\circ}$. We used a Levenberg-Marquadt method (Press et al. 1992) with multiple starting configurations to produce goodness-of-fit values for different numbers of subpulses. By comparing these $\chi^{2}$ values, we located the subpulses that had high significance levels.

Upon finding these normal to strong subpulses, we set out to detect the weaker ones over the noise. For this purpose we composed driftbands out of the individual subpulses already identified. On a one-by-one basis, a subpulse was either added to a path that had predicted its position to within $P_{2} / 4$ ( $P_{2}$ being the average separation between two subpulses within a single pulse, about $11^{\circ}$ longitude), or taken to be the start of a new path. Paths ended if no subpulse fitted the path for more than 5 pulses, or upon reaching a null. On the first run, only paths longer than ten pulses were allowed to survive, so as to eliminate the interference of short run-away paths. This allowed a steady pattern of long paths to grow, incorporating about $90 \%$ of the subpulses found. On the second run, paths longer than 5 pulses were allowed to form: these shorter paths occur only around nulls, where the normal, long paths are interrupted.

The drifting pattern thus formed predicted the locations of the weaker subpulses. Around each predicted position, we isolated a section of the data. This section was taken as large as possible without interfering with other, previously fitted subpulses. We checked the significance of fitting a single Gaussian to indicate the presence of a subpulse.

In the same way as described above, the final driftpattern was then identified using the extended set of subpulses.

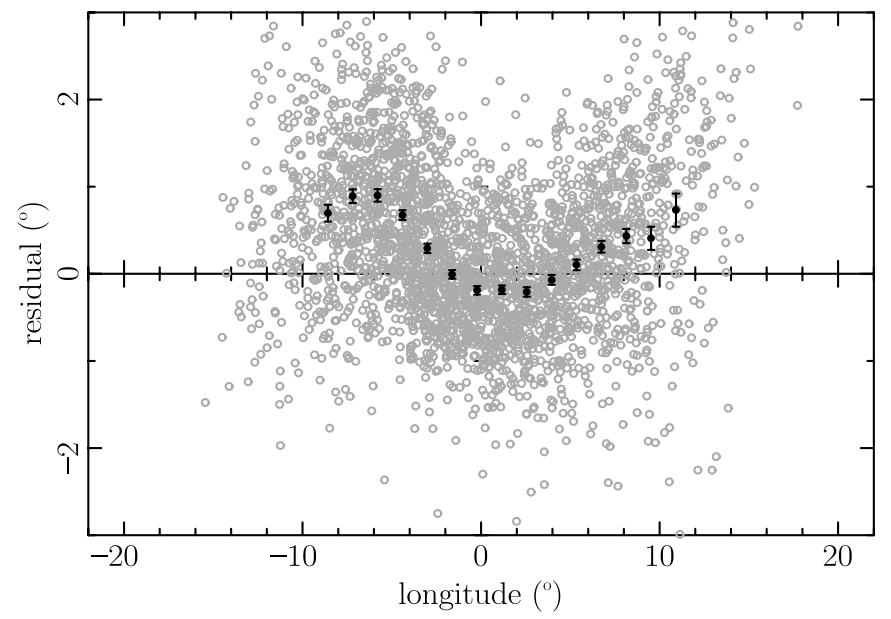

Fig. 3. Horizontal residuals to straight line fits to the driftbands, individual and binned, for 2000 pulses.

To check the method, we compared the observed average profile with the one recreated from the Gaussians fits to the subpulses. From their match, we concluded that the fitting procedure was effective.

\section{Results}

\subsection{Driftband fitting}

The driftbands are not straight, but show a systematic curvature (Davies et al. 1984). This is seen most strikingly when we plot the residuals to straight line fits: Fig. 3 shows the longitudinal offset between the actual and predicted positions of the subpulses. Near the edges of the profile the subpulses arrive later than is to be expected based on a linear driftband, in the middle they arrive sooner.

This probably explains the results of Popov \& Smirnova (1982). After fitting straight lines to all driftbands, they find that the driftrate of the last driftband before the null is $20 \%$ higher than that of a normal driftband. As seen in the curvature of the driftbands, however, a normal driftband consists of a fast drifting first half and a slow drifting second half. If a driftband is cut by a null, the part before the null consists only of the fast drifting first half, resulting in a higher average driftrate over this shorter driftband.

To make our results independent of the longitude, the non-linearity of the driftbands is taken into account in all the following driftrate calculations. In these cases, the positions of individual subpulses are corrected by subtracting the appropriate residual value.

\subsection{Nulling}

\subsubsection{Shortest nulls}

The main criterion used to decide whether or not to include certain datasets was the complete separation of the on-pulse and off-pulse energy distributions. Therefore, the set of null identified is genuine and complete. This allows 


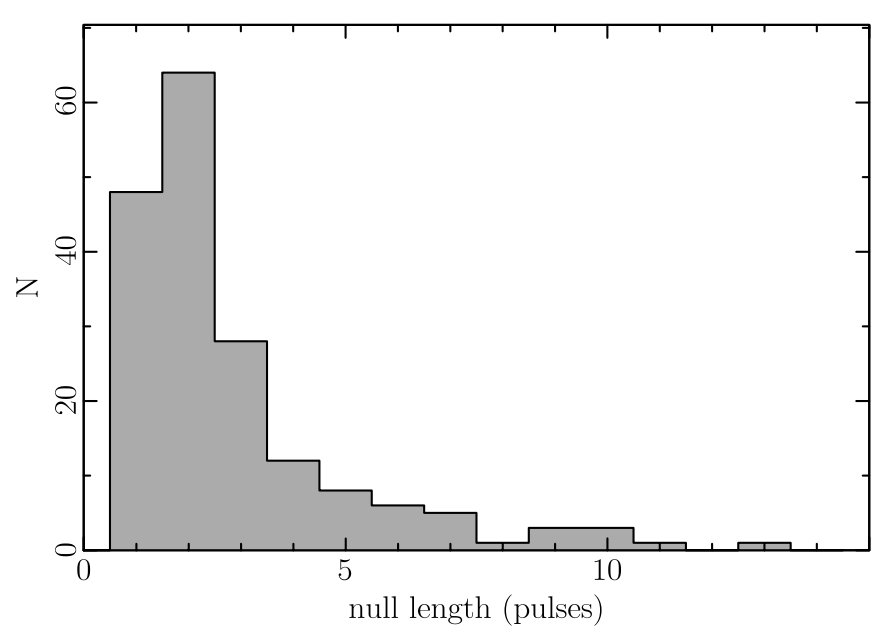

Fig. 4. Length histogram for the 180 nulls.

us to investigate the underlying statistics of null occurrence and length, and estimate the influence of short nulls.

The chance of finding $0809+74$ in the null state is on average about $1.4 \%$. If the null lengths were distributed in a Poissonian fashion, we would find a preponderance of one-pulse nulls and very few longer ones. However, in the null-length histogram (Fig. 4) we find a peak at nulls of length 2, a significant decrease towards shorter lengths and a considerable number of long nulls, showing that the occurrence of nulls is not governed by pure chance.

Comparing this histogram with the one previously found by Lyne \& Ashworth (1983), we see that we identify about twice as many one-pulse nulls in the data. Still, many nulls shorter than one period must pass unnoticed, as they occur when the pulsar faces away from us.

We know that nulls, especially long ones, have a distinct impact on the drifting pattern in their vicinity. Although the impact of shorter nulls is less, a large number of unnoticeable, short nulls $\left(<P_{1}\right)$ might seriously influence the drifting pattern we are trying to understand.

Using the null-length histogram we can estimate the number of these short nulls. It peaks at two-pulse nulls, and the distribution decreases towards shorter lengths. Assuming that the underlying distribution of null-lengths is continuous, this tendency of decreasing occurrence towards shorter nulls implies that there is a small number of nulls shorter than one pulse period. Extrapolating the decrease leads to an estimate of about 15 unnoticeable short nulls in the null-length interval from 0 to 0.5 pulses.

The low frequency of their occurrence (0.04\%) indicates the influence of short nulls on the drifting pattern is negligible.

\subsubsection{Null versus burst length}

The next question we address involves the interval between adjacent nulls (the so-called burst) and the duration of the nulls. Does waiting longer for a null mean it will last longer, too? We have checked these relationships and have found that the lengths of neighbouring nulls and bursts are independent.

\subsubsection{Position jump over nulls}

If the nulling mechanism is independent of the position of the subpulses, we expect that the distribution of subpulse positions is the same for the normal pulses and the pulses that immediately precede a null. We find no proof of differences in these distributions and conclude that there is no preference for a null to start at a certain subpulse position.

The positions of subpulses change over a null. We derived this shift of the subpulse pattern for each null in our sample. As the positions of the individual subpulses were already identified, this shift was simply extracted. Each subpulse before the null matched a subpulse after the null, if the latter fell within $-3 P_{2} / 4$ to $P_{2} / 4$ of the former. This range is symmetric around the average expected jump over a null, so as to minimise the number of ambiguous cases. In the following analysis, we used the average of all individual subpulse shifts within one pulse.

Figure 5 shows this shift in the subpulse position over a null, corrected for the non-linear behaviour of the driftband. If the motion of the subpulses were independent of the emission, the subpulses would continue to drift invisibly throughout the null state, and reappear at a very different position. The associated shift in positions would then be spread around the diagonal dashed line in Fig. 5.

If, on the other hand, the subpulse drift would cease abruptly and completely during the null, the jump in position would be between zero and two times the average shift in longitude between normal pulses. The uncertainty in this estimate arises from the fact that neither the start

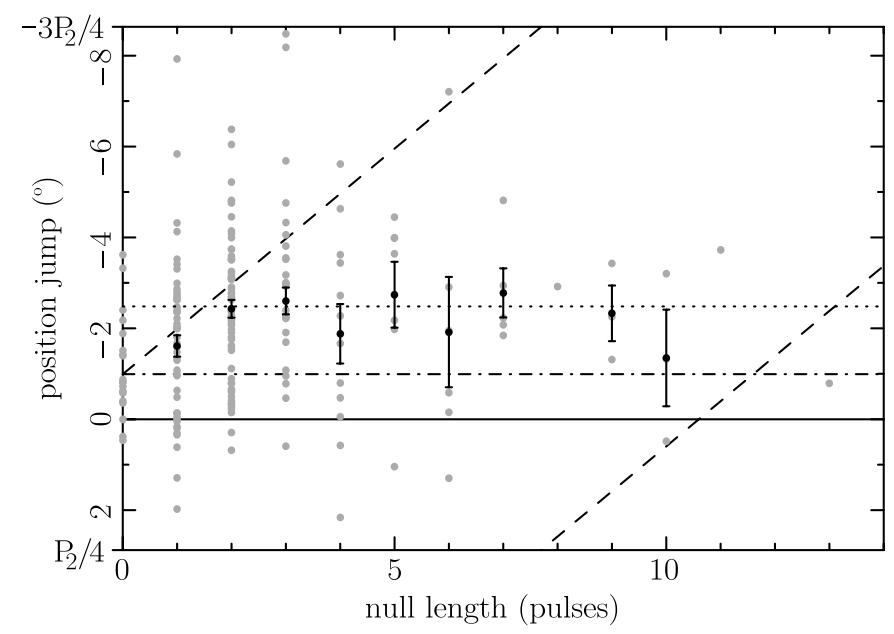

Fig. 5. Jump in subpulse position against null length. The bottom of the plot falls at $P_{2} / 4$, the top at $-3 P_{2} / 4$. The diagonal dashed line is the predicted subpulse path if the drifting were independent of the nulls. The horizontal dash-dotted line is the predicted path for a sudden and complete stop of drifting during the null. The horizontal dotted line is the average jump found for the nulls longer than 1 period. 


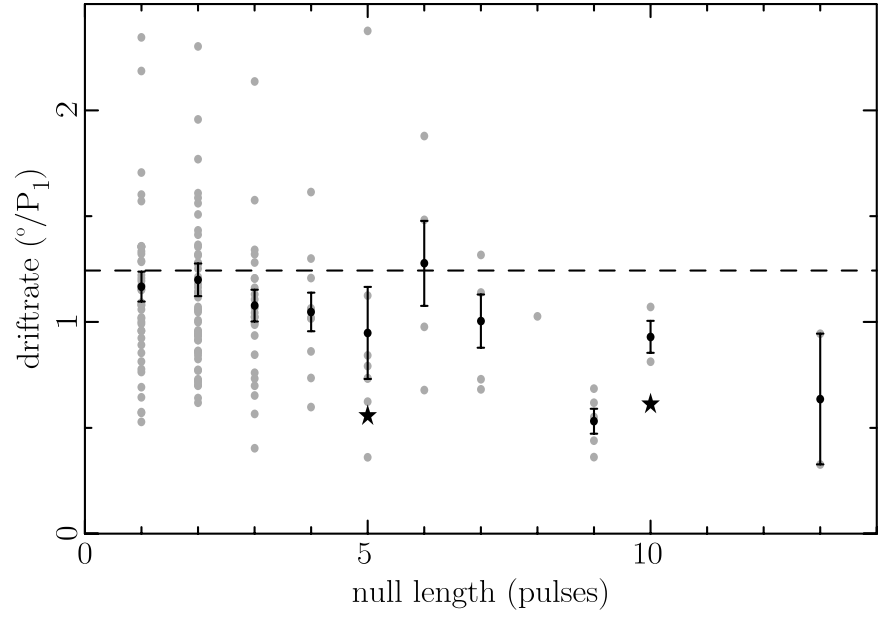

Fig. 6. Drift after the null versus the length of the null. We show the average driftrate of the first 6 pulses for each driftband after a null (gray points). The average per null length (black points) with its error is also plotted. The normal driftrate, corrected for the driftband curvature, is indicated by the horizontal dashed line. The stars denote the driftrate for the slow drifting mode sequences. One pulse often consists of more than one subpulse, so after many nulls we see several driftbands reappear. The number of driftrates plotted here is therefore larger than the total number of nulls.

nor end of the null are known more accurately than to one pulse period. The mean jump in position over the null would then follow the horizontal dash-dotted line in Fig. 5.

After discarding the three ambiguous cases (points near the top edge of Fig. 5), we computed averages for each null length. These averages follow neither of the two cases outlined above. There is too much change in position over the null to be accounted for by just an abrupt stop of the drift, and there is no evidence for steady (albeit slower than regular) drift during the null.

We do see that for nulls longer than one, the jump is constant, $1.47 \pm 0.16^{\circ}$ above the offset value that we would expect in the case of no drift. This independence of null length and subpulse jump over the null is shown as the dotted horizontal line in Fig. 5.

\subsubsection{Driftrate around the null}

We have computed the driftband slope around nulls, correcting for their general curvature.

For the driftrate before the null, we fitted straight lines to the last six subpulses of each driftband the ended with the null. We find that this driftrate just before a null does not deviate from the normal driftrate.

The driftrate after nulls is different from the normal driftrate, though. Although there is some spread, all the driftrates we find after longer nulls are lower than the normal average value (Fig. 6).

\subsubsection{Average pulse profile around null}

To investigate whether the change from normal emission to the null state is sudden or more gradual, Lyne \& Ashworth (1983) compared the energies of the pulses near a null. The finite chance that the emission from the pulsars drops or rises within the pulse window would influence the brightness of the pulses around the null. The last pulse before the null was indeed found to be less bright than a normal pulse. The first pulse after the null, however, was considerably more bright than a normal pulse.

We have not just looked at the energies of these neighbouring pulses, but also at their profiles. To this end, we have averaged all the last pulses before nulls longer than 1 period, and all the first pulses after these nulls. The results, shown in Fig. 7 and condensed in Table 1, are surprising. Not only do we find the expected differences in brightness, we also see a significant offset in the pulse position for the first pulses after nulls.

\subsection{Slow drifting mode}

Although we had set out to quantify the normally very regular drifting behaviour of $0809+74$, we unexpectedly found two occasions in which the pulsar clearly deviates from its normal drifting mode. We will refer to these sequences by the year in which they were observed, 1999 and 2000. In Fig. 8a we have plotted the derived longitude (Lyne \& Ashworth 1983) of subsequent pulses around the mode changes. The derived longitude of the subpulses effectively converts the different short driftbands into one long one. Figure 9 shows a grayscale plot of the two slow drifting series.

The most striking difference, as the drifting pattern is concerned, is clearly the decrease of the driftrate by about $50 \%$.

We see that in both the observations, the drifting mode changes during or immediately following a long null. After

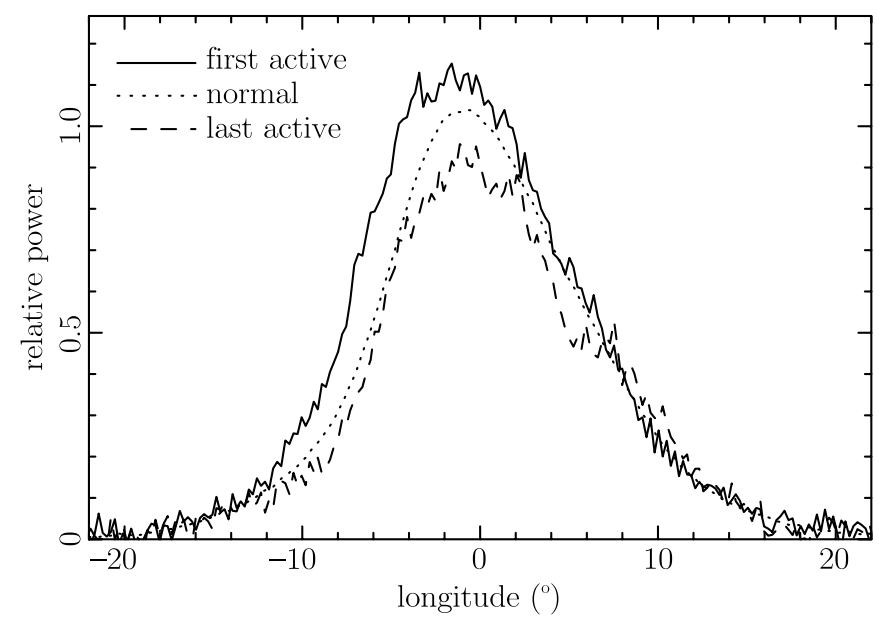

Fig. 7. Average profiles for the pulses adjacent to nulls, for all 131 nulls longer than 1 pulse. Shown are: the first active pulse after the null, the last active pulse before the null and, for reference, the normal average profile. 


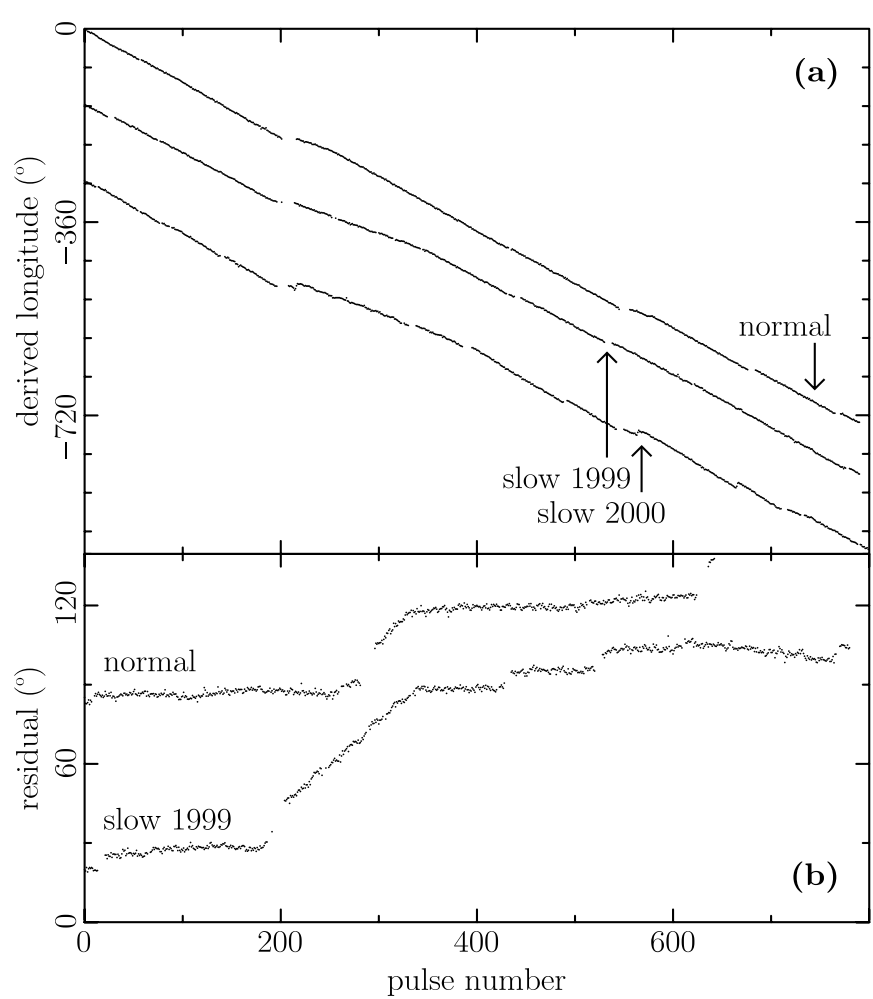

Fig. 8. a) Derived longitudes of three pulse sequences. The gaps in the curves are nulls. The sequences are aligned on the ends of the long nulls around pulse 200. The top line shows the normal drifting behaviour around nulls of lengths 13, 9, 3 and 2, respectively. The middle line shows the 1999 slow drifting sequence (offset $-150^{\circ}$ ), the bottom line shows the 2000 sequence (of fset $-300^{\circ}$ ). b) Deviation of the derived longitude from normal drifting for the 1999 and the normal sequence shown in panel a). The transitions from slow to normal drifting have been aligned.

these nulls of length 10 and 5 respectively, almost all drifting properties reappear at new values, as laid out in Table 2. As some of these properties normally already show change over time, we compare the slow drifting sequences (labeled "slow") to the 600 pulses that surround them (labeled "normal").

In the first three columns of Table 2, we investigate the parameters of the individual subpulses in both modes. Immediately we see a very interesting change in the relative average position of the subpulses. Right after the null, there is a definite shift in position towards earlier arrival, that can already be seen in a plot like Fig. 9. Interestingly, this shift of the pulse window is similar to the average jump in subpulse position we see over a normal null.

In the slow drifting mode, the subpulses are slightly wider, but their heights remain the same.

Next, we explore the driftband characteristics in the last three columns of Table 2 . The average longitude separation of two adjacent driftbands, $P_{2}$, decreases significantly by about $15 \%$. Hence, in the slow drifting mode the subpulses within each pulse are spaced closer together than normal.
When we compare the driftrates of the subpulses between the two modes, we find that the driftrate in the slow drifting mode is almost halved. This puts the new driftrate right in the range of driftrates we usually find after a longer null. We have indicated these driftrate values with black stars in Fig. 6 .

Being dependent on $P_{2}$ and the driftrate, the fractional change in $P_{3}$ (the recurrence time of a driftband) is comparably large.

In both cases the new drifting mode is stable for about 120 pulses and then changes back to normal. To illustrate this change, we have plotted the deviation of the derived longitude from normal drifting in Fig. 8b. For the 1999 observation, we see a normal driftrate up to the null at pulse 200. After this null, the driftrate is smaller, up to pulse 330. In about 20 pulses, the drift then speeds up back to its normal value.

For reference, we have also plotted the drifting behaviour after the longest null in our sample. Again, we see a speedup to the normal driftrate in about 20 pulses at pulse 330, very similar to the slow drifting speedup time scale.

The slow drifting interval in the 2000 observation (right panel of Fig. 9) is followed and stopped by a series of frequent, longer than average nulls, out of which the pulsar emerges in its normal drifting pattern.

These surprising changes in subpulse positions, widths and separations must influence the average pulse profile during the slow drifting mode. Noting the many similarities between slow drifting pulses and the pulses that follow nulls (halved driftrate, similar offsets, similar speedup) we are interested in the possible similarities between the average profiles of these pulses, especially since the average profile of the pulses after the null is singularly bright and shifted in longitude.

In Fig. 10 and Table 1 we compare the pulsar's normal average profile with the average profile in the slow drifting

Table 1. Comparison of the pulsar's average emission profiles for different sets of pulses. We show the height, full width at half maximum (fwhm) and position of the Gaussians that fitted the profiles best. The "normal" subset consist of all non-null pulses in the dataset. All heights and positions in this table are relative to the height and position of this "normal" set. The set labeled "last active" contains all 131 pulses that preceded a null that was longer than 1 pulse period. The characteristics of the pulses that followed these nulls are labeled "first active". The subsets "slow 1999" and "slow 2000" contain the 120 pulses that form the slow drifting sequences observed in 1999 and 2000, respectively.

\begin{tabular}{lccc}
\hline \hline & height & position $\left({ }^{\circ}\right)$ & fwhm $\left({ }^{\circ}\right)$ \\
\hline normal & $1.000 \pm 0.003$ & $0.000 \pm 0.016$ & $13.33 \pm 0.04$ \\
last active & $0.875 \pm 0.005$ & $0.27 \pm 0.12$ & $13.96 \pm 0.09$ \\
first active & $1.112 \pm 0.005$ & $-0.77 \pm 0.12$ & $13.84 \pm 0.08$ \\
\hline slow 1999 & $1.272 \pm 0.005$ & $-1.51 \pm 0.3$ & $11.99 \pm 0.06$ \\
slow 2000 & $1.311 \pm 0.008$ & $-0.75 \pm 0.3$ & $10.52 \pm 0.08$ \\
\hline
\end{tabular}




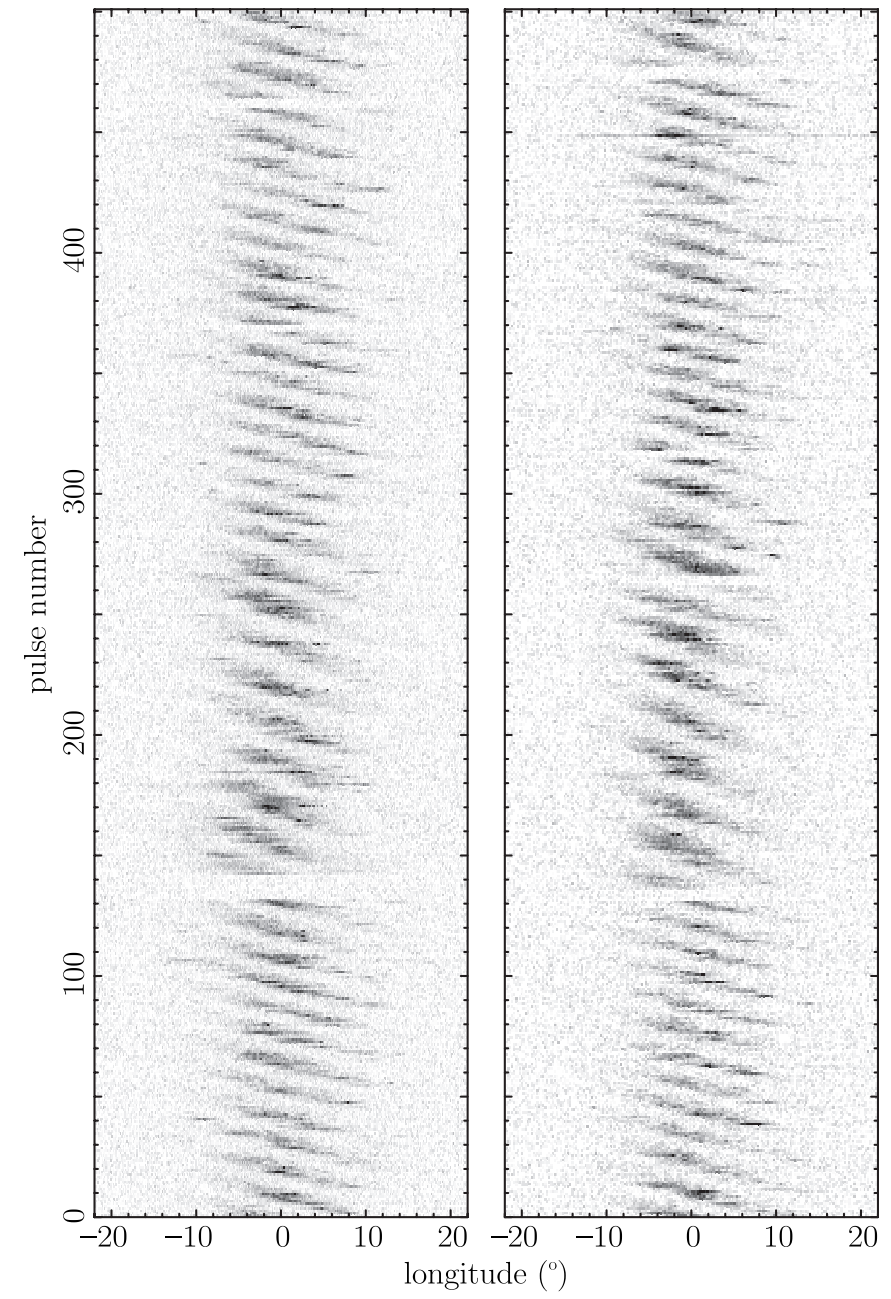

Fig. 9. Grayscale plots of the pulses composing the two slow drifting sequences. The grayscale linearly depicts the intensity of a sample. The left series was observed in 1999, the right series in 2000. After a null of length 10 (1999 series) and 5 (2000 series) around pulse number 140, the driftrate and driftband separation are steadily different for about 120 pulses. From pulse 260 on, the drifting changes back to normal.

mode. In both observations, the slow drifting mode average profile is much brighter than the normal profile and offset towards earlier arrival - exactly the two peculiarities we found in the average pulse profile after a null as well.

\section{Discussion}

\subsection{Driftband fitting}

The non-linearity of the driftbands was already noted by Page (1973), who integrated several hundreds of pulses to compare the shape of the driftbands in different observations. He found considerable differences between these driftband shapes. We compare the average drift path over several thousands of pulses however, and find that the curvature of the driftbands remains the same.

The curvature of the driftband can be a direct consequence of the curved geometry of the emission region (Krishnamohan 1980). In that case, the shape of the drift-

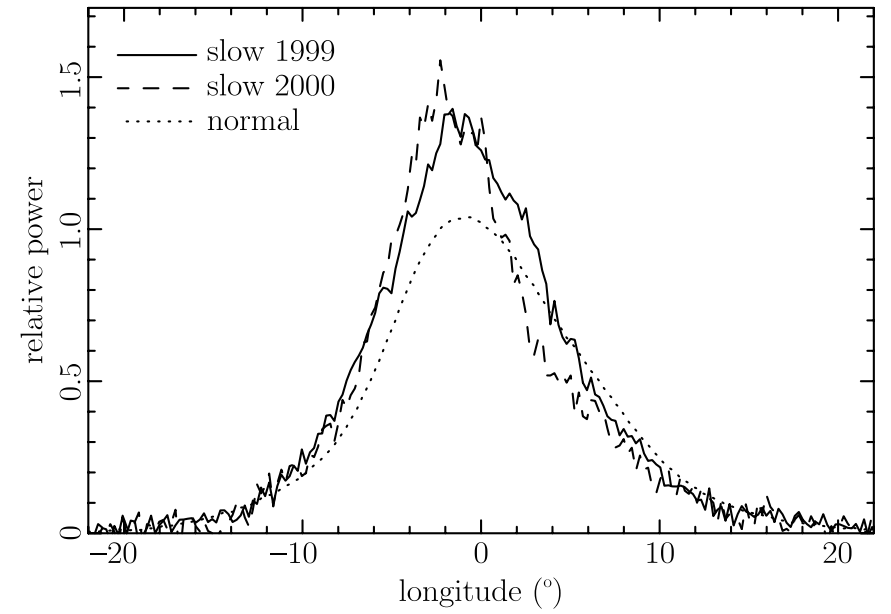

Fig. 10. Average profiles for the slow drifting mode sequences. The "slow 1999" and "slow 2000" profiles are averaged over the 120 pulses that composed the slow drifting sequences observed in 1999 and 2000, respectively. The normal average profile is plotted for comparison.

band is expected to be point-symmetric: the curve will then be unchanged after mirroring it in both axes. This point-symmetry would then return in the residuals to straight line fits (Fig. 3). The shape of the driftbands we find, however, is not point-symmetric but axisymmetric: the curve is unchanged after mirroring in the y-axis.

We could still tie the curvature of the driftbands to the emission-region geometry, by assuming that part of the pulse profile of this pulsar is missing. In that case, the driftband shape we find would represent only part of the total expected shape.

This suggestion that part of the profile of $0809+74$ is missing fits in with observations of this pulsar's pulse shape at different frequencies (Bartel et al. 1981; Kuzmin et al. 1998). These imply that at lower frequencies the profile is partially "absorbed".

\subsection{Shortest nulls}

We find that the number of invisible nulls is low. That means we can study of the drifting properties of $0809+74$ straightforwardly.

\subsection{Slow drifting mode and nulling}

With our investigation of $0809+74$ still ongoing, we discuss here only the phenomena themselves and defer their interpretation to a subsequent paper.

The similarity between this pulsar's behaviour in the slow drifting mode and around nulls is striking:

- The slow drifting sequences start at nulls.

- The driftrate of the slow drifting mode is a lower limit to the driftrates found after all nulls.

- The speedup from slow drifting to normal is identical to the speedup after a null. 
Table 2. Subpulse and driftband properties for the slow drifting mode sequences and the surrounding normal drifting pattern.

\begin{tabular}{|l|c|c|c|c|c|c|}
\hline \hline & \multicolumn{3}{|c|}{ averages from Gaussian fits to subpulses } & \multicolumn{3}{|c|}{ averages from straight line fits to driftbands } \\
\cline { 2 - 7 } & relative height & relative position $\left(^{\circ}\right)$ & $f w h m\left(^{\circ}\right)$ & $P_{2}\left(^{\circ}\right)$ & driftrate $\left({ }^{\circ} / P_{1}\right)$ & $P_{3}\left(P_{1}\right)$ \\
\hline normal 1999 & $1.00 \pm 0.02$ & $0.00 \pm 0.11$ & $5.39 \pm 0.06$ & $11.61 \pm 0.14$ & $1.086 \pm 0.011$ & $10.72 \pm 0.13$ \\
slow 1999 & $1.06 \pm 0.05$ & $-1.2 \pm 0.3$ & $5.75 \pm 0.11$ & $9.9 \pm 0.5$ & $0.606 \pm 0.019$ & $16.5 \pm 0.5$ \\
\hline normal 2000 & $1.00 \pm 0.02$ & $0.0 \pm 0.2$ & $5.22 \pm 0.06$ & $11.33 \pm 0.14$ & $1.043 \pm 0.014$ & $10.88 \pm 0.13$ \\
slow 2000 & $0.99 \pm 0.04$ & $-1.8 \pm 0.2$ & $5.55 \pm 0.11$ & $9.63 \pm 0.3$ & $0.54 \pm 0.02$ & $17.6 \pm 0.5$ \\
\hline
\end{tabular}

- The average profiles of both the post-null and slow drifting pulses are brighter than normal.

- These average profiles are both displaced to earlier arrival.

- This displacement of the average profile is caused by offsets in the individual subpulses.

- These offsets are identical to the jump of the subpulses over the null.

The natural conclusion is that the behaviour after a null and the slow drifting mode are the same, quasi-stable phenomenon. Normally the pulsar reappears from the null in the slow drifting mode. After a variable time, it quickly evolves to the normal mode. Therefore, right after a normal null we see either a short sequence of slow drifting, or the transition back to normal drifting. In the case of the long slow drifting mode sequences, the metamorphosis back to the normal mode is delayed.

This would explain all the similarities we found above. After a normal null, the pulsar is in the slow drifting mode or changing back to the normal mode. All the characteristics of the slow drifting mode can then be identified in the post-null behaviour, although they will be less pronounced; the transition to normal drifting may already be taking place.

When the driftrate increases at the end of a slow drifting sequence, this speedup is quick and identical to the speedup seen after a normal null (see Fig. 8b).

The post-null driftrate values are found in between the slow drifting and normal driftrate value, depending on how soon the transition back to normal takes place (see Fig. 6). Although the return from the low to the normal driftrate will be quick, the time the driftrate is low may vary for different nulls of the same length. The average of these different sequences will then resemble the slow exponential decay found by Lyne \& Ashworth (1983).

Comparing average pulse profiles, the increased brightness and the pulse offset of the post-null average profile are attenuated versions of similar deviations seen in the slow drifting mode profile. (see Table 1, Figs. 7 and 10).

The average profile offset we see in the slow drifting mode is caused by a shift of the window in which the subpulses appear (Tables 2 and 1).

The change in position of the post-null average pulse profile must then be caused by this shift of the pulse window as well. The magnitude of this shift is identical to the subpulse-longitude jump over normal nulls.
This means that the subpulse-position jump over a null is caused by a displacement of the pulse window as a whole, like in the slow drifting mode.

Previously, this jump was thought to be the effect of the subpulse-drift speedup during the null. For this speedup to produce the observed jump in subpulse position, the time scale involved had to be long, contrasting the short time scales found for the slowdown of the subpulse drift and the rise and fall of the emission around a null.

With the displacement of the subpulses over the null accounted for, the estimated speedup time of the subpulse drifting is negligible: the preservation of the position of the subpulses over the null now only allows for a quick speedup of the subpulse drift, putting all time scales of emission and drift rise and decay around the nulls in the same range.

\section{Conclusions}

After many or all nulls, $0809+74$ emits in a mode different from the normal one. This mode is quasi-stable, normally changing back to normal in several pulses. This is seen as the normal behaviour after a null, of which the reduced driftrate is the most striking characteristic. Occasionally, the quasi-stable slow drifting configuration persists for over a hundred pulses before changing back to the normal mode.

The pulses in the slow drifting mode and consequently all pulses after the null are brighter than normal pulses. In the slow drifting mode, the subpulses are closer together, they drift more slowly through the profile and the window in which they appear is offset towards earlier arrival.

This offset of the pulse window accounts for the displacement of subpulses over the null. When taking this shift of the window into account, we find that the longitude of the subpulses is perfectly conserved over a null. This indicates that the speedup time for the subpulse drift is short.

\section{References}

Alexeev, Y., Vitkevich, V., \& Shitov, Y. 1969, Astron. Circ. Acad. of Sci. USSR, N495, 4

Bartel, N., Kardashev, N. S., Kuzmin, A. D., et al. 1981, A\&A, 93, 85

Cole, T. W. 1970, Nature, 227, 788 
Davies, J. G., Lyne, A. G., G., S. F., et al. 1984, MNRAS, 211, 57

Deshpande, A. A., \& Rankin, J. M. 1999, ApJ, 524, 1008

Deshpande, A. A., \& Rankin, J. M. 2001, MNRAS, 322, 438

Drake, F. D., \& Craft, H. D. 1968, Nature, 220, 231

Filippenko, A. V., \& Radhakrishnan, V. 1982, ApJ, 263, 828

Goldreich, P., \& Julian, W. H. 1969, ApJ, 157, 869

Kouwenhoven, M. L. A. 2000, Ph.D. Thesis, Utrecht University

Krishnamohan, S. 1980, MNRAS, 191, 237

Kuzmin, A. D., Izvekova, V. A., Shitov, Y. P., et al. 1998, A\&AS, 127, 255

Lyne, A. G., \& Ashworth, M. 1983, MNRAS, 204, 519

Page, C. G. 1973, MNRAS, 163, 29

Popov, M. V., \& Smirnova, T. V. 1982, Sov. Astron., 26, 439
Press, W. H., Teukolsky, S. A., Vetterling, W. T., \& Flannery, B. P. 1992, Numerical Recipes: The Art of Scientific Computing, 2nd edition (Cambridge: Cambridge University Press)

Ritchings, R. T. 1976, MNRAS, 176, 249

Ruderman, M. A., \& Sutherland, P. G. 1975, ApJ, 196, 51

Sturrock, P. A. 1971, ApJ, 164, 529

Taylor, J. H., \& Huguenin, G. R. 1971, ApJ, 167, 273

Unwin, S. C., Readhead, A. C. S., Wilkinson, P. N., \& Ewing, M. S. 1978, MNRAS, 182, 711

Vitkevich, V., \& Shitov, Y. 1970, Nature, 225, 248

Voûte, J. L. L. 2001, Ph.D. Thesis, University of Amsterdam

Voûte, J. L. L., Kouwenhoven, M. L. A., van Haren, P. C., et al. 2002, A\&A, accepted 